\title{
Fracture permeability and seismic wave scattering -Poroelastic Linear-Slip Interface model for heterogeneous fractures
}

\author{
Seiji Nakagawa and Larry R. Myer, Lawrence Berkeley National Laboratory, Berkeley, CA, USA
}

\section{Summary}

Schoenberg's Linear-slip Interface (LSI) model for single, compliant, viscoelastic fractures has been extended to poroelastic fractures for predicting seismic wave scattering. However, this extended model results in no impact of the in-plane fracture permeability on the scattering. Recently, we proposed a variant of the LSI model considering the heterogeneity in the in-plane fracture properties. This modified model considers wave-induced, fracture-parallel fluid flow induced by passing seismic waves. The research discussed in this paper applies this new LSI model to heterogeneous fractures to examine when and how the permeability of a fracture is reflected in the scattering of seismic waves. From numerical simulations, we conclude that the heterogeneity in the fracture properties is essential for the scattering of seismic waves to be sensitive to the permeability of a fracture.

\section{Introduction}

Schoenberg originally formulated the Linear-Slip Interface (LSI) model for predicting the frequency- and compliance-dependent scattering of seismic waves, by a flat, two-dimensional fracture (Schoenberg, 1980). This original model is for a viscoelastic fracture and a background. The applicability of the model has been demonstrated by many laboratory experiments (e.g., Pyrak-Nolte et al. 1990; Myer et al., 1990; Hsu and Schoenberg, 1993).

For examining the effect of pore fluids, poroelastic version of the models have been developed, for open fractures (Bakulin and Molotkov, 1997) and fractures clogged with gouge materials with a range of permeability (Nakagawa and Schoenberg, 2007). These poroelastic LSI models, however, predicted that the scattering of seismic waves are not affected by the in-plane (or fracture-parallel) permeability of a fracture, because no permeabililty terms appear in the models (Note that the "clogged-fracture" model predicts dependency on the out-of-plane [or fracturenormal] permeability of the fracture, owing to the gouge layer.)

The primary reason for this apparent lack of sensitivity to permeability may be that these poroelastic LSI models assume a fracture with a homogeneous distribution of fracture properties including mechanical compliance, width (opening), fluid properties, and permeability. Heterogeneous distributions of these properties can result in increased local gradients of waveinduced fluid pressures, which can enhance the fluid flow within the fracture. (e.g., Dvorkin et al., 1994; Pride and Berryman, 2003ab) 


\section{Poroelastic Heterogeneous Fracture Model}

Recently, we developed a new poroelastic LSI model including the fracture-parallel permeability, considering the heterogeneity of a fracture (Nakagawa, 2009). In the following, we will first review this poroelastic LSI model. Using this model, numerical examples of seismic wave reflection will be given for one-dimensional fractures with a range of permeability. A particular focus will be on the differences in the fast $\mathrm{P}$ wave scattering for water and supercritical $\mathrm{CO}_{2}$-saturated fractures.

\section{Poroelastic LSI Model for Heterogeneous Fractures}

The primary assumption of the LSI models is that the local scales of a fracture- - such as fracture asperity height, contacting patch diameter and spacing - are much smaller than the wavelengths. This allows us to neglect inertia-related quantities, resulting in simple, quasi-static relationships between wave-induced displacement and stress across a fracture.

We assume a flat, infinite fracture with possible partial contacts between the two surfaces. On this fracture, we assume a Cartesian coordinate system with the 1,2 plane aligned with the fracture plane. For simplicity, we also assume that the flow and mechanical property of the fracture is rotationally invariant around the 3 axis.

First, continuity conditions for stress and fluid pressure are given by:

$$
\left[\tau_{13}\right]=0,\left[\tau_{23}\right]=0,\left[\tau_{33}\right]=0,\left[-p_{f}\right]=0
$$

The square brackets indicate the difference in the related quantity (two shear and one normal total stresses $\tau_{i 3}, i=1,2,3$, and fluid pressure $-p_{f}$ in the above equations) across a fracture. Next, the constitutive relationships between solid displacement jumps (linear slips) and stress and pressure are given as:

$$
\begin{aligned}
& {\left[u_{1}\right]=\eta_{T} \tau_{13}} \\
& {\left[u_{2}\right]=\eta_{T} \tau_{23}} \\
& {\left[u_{3}\right]=\eta_{N d}\left\{\tau_{33}-\alpha\left(-p_{f}\right)\right\}}
\end{aligned},
$$

where $\eta_{T}$ is the shear compliance, $\eta_{N d}$ is the dry (or drained) normal compliance of a fracture. The third equation indicates the effective stress law, which includes the Biot-Willis coefficient $\alpha$.

The final condition to be specified is for a jump in the fluid flux displacement $\left[w_{3}\right]$ in the fracture-normal direction (fluid exiting the fracture). We realize that this can be viewed as a fluid mass or volume conservation relationship, which is given by:

$$
\left[w_{3}\right]=-\alpha\left[u_{3}\right]+\eta_{M}\left(-p_{f}\right)+F .
$$




\section{Poroelastic Heterogeneous Fracture Model}

The first term in the right hand side is for the fluid squeezed out due to the closure of a fracture; the second term is for the expansion of fluid and minerals within a fracture (therefore, this includes the fluid/mineral compliance $\eta_{M}$ defined by Nakagawa and Schoenberg, 2007); and the third term $F$ is for the influx of fluid by the fracture-parallel flow, which should be given by:

$$
F \equiv-\nabla^{(2)} \cdot\left(\overline{\mathbf{w}}^{(2)} h_{H}\right)=-\partial_{1}\left(\bar{w}_{1} h_{H}\right)-\partial_{2}\left(\bar{w}_{2} h_{H}\right) .
$$

$h_{H}$ is the local hydraulic width of the fracture. The average flow displacement $\bar{w}_{i}(i=1,2)$ in the fracture is related to the fluid pressure via

$$
\overline{\dot{w}}_{i}=(-i \omega) \bar{w}_{i}=\frac{k_{i j}(\omega)}{\eta_{f}}\left\{\frac{\partial\left(-p_{f}\right)}{\partial x_{j}}-\rho_{f} \bar{u}_{j}\right\}
$$

where $k_{i j}(\omega)$ is the frequency-dependent permeability of the fracture, $\eta_{f}$ is the fluid viscosity, $\rho_{f}$ is the fluid density, and $\overline{\ddot{u}}_{j}$ is the averaged acceleration of fracture surfaces. This acceleration term is generally small and can be ignored, as other intertia-related terms in the LSI model (quasistatic model). Using these equations, the LSI model for a poroelastic, heterogeneous fracture is obtained as:

$$
\begin{aligned}
& \boldsymbol{\tau}^{+}\left(x_{1}, x_{2}\right)=\boldsymbol{\tau}^{-}\left(x_{1}, x_{2}\right), \\
& \mathbf{u}^{+}\left(x_{1}, x_{2}\right)-\mathbf{u}^{-}\left(x_{1}, x_{2}\right)=\boldsymbol{\eta}\left(x_{1}, x_{2}\right) \boldsymbol{\tau}^{+}\left(x_{1}, x_{2}\right),
\end{aligned}
$$

where the superscripts " +" and "_" indicate the opposite sides of the fracture, and

$$
\begin{aligned}
& \boldsymbol{\eta} \equiv\left[\begin{array}{cccc}
\eta_{T} & & & \\
& \eta_{T} & & \\
& & \eta_{N d} & -\alpha \eta_{N d} \\
& & -\alpha \eta_{N d} & \alpha^{2} \eta_{N d}+\eta_{M}+\frac{1}{i \omega} \nabla^{(2)} \cdot\left(\varsigma(\omega) \nabla^{(2)}\right)
\end{array}\right] \\
& \boldsymbol{\tau}^{ \pm} \equiv\left[\begin{array}{llll}
\tau_{31} & \tau_{32} & \tau_{33} & -p_{f}
\end{array}\right]^{T}\left(x_{3} \rightarrow \pm 0\right), \\
& \mathbf{u}^{ \pm} \equiv\left[\begin{array}{llll}
u_{1} & u_{2} & u_{3} & w_{3}
\end{array}\right]^{T}\left(x_{3} \rightarrow \pm 0\right), \\
& \boldsymbol{\varsigma}(\omega) \equiv \mathbf{k}(\omega) h_{H} / \eta_{f}, \mathbf{k}(\omega) \equiv\left[k_{i j}(\omega)\right] \text {. }
\end{aligned}
$$

The two-dimensional gradient operators $\nabla^{(2)}$, in the $(4,4)$ term in the compliance matrix $\boldsymbol{\eta}$, indicates that the above matrix equation involves first and second derivatives of fluid pressure. Previously, for a thin, homogeneous fracture, the $F$ term was neglected because it is of order $O(h)$ which vanishes for small fracture thickness $h$ ( hydraulic fracture thickness $h_{H}$ ). However, heterogeneity in the fracture properties may result in very large gradients in both fluid pressure 


\section{Poroelastic Heterogeneous Fracture Model}

and the permissivity tensor $\varsigma(\omega)$, which increases the overall magnitude of the $F$ term for a small but finite $h_{H}$. In the following, we will examine some examples of heterogeneous fractures when this is the case.

\section{D Simulations}

The boundary conditions in equations (6)-(11) can be implemented in a finite difference model or a finite element model for computing seismic wave scattering by a fracture with an arbitrary distribution of fracture properties (e.g., Coates and Schoenberg, 1995). Here, we will use an alternative technique based upon wavenumber-domain integration, proposed by Nakagawa et al. (2004). The application of this technique for poroelastic fractures was shown by Nakagawa (2009) and will not be discussed here.

\section{$1 D$ Fracture models}

In the following examples, we will assume a flat, one-dimensional fracture with periodic fracture (hydraulic) width and compliance distributions shown in Figure 1. The background material properties are assumed to be those typical for medium-porosity sandstone (e.g., Berea), except two extreme cases of low and high permeability are examined (10 $\mu \mathrm{D}$ and $10 \mathrm{D})$. (Note: The dry normal and shear compliance values are assumed to be equal, and they are proportional to the hydraulic fracture width.) For examining the effect of fracture permeability on seismic wave scattering, the local fracture permeability, which is a function of the local fracture width, is specified using the Biot's dynamic permeability for a flat, open, parallel channel (Biot, 1956) multiplied with a range of reduction factors $R(=1 / 1000,1 / 100,1 / 10$, and 1). Therefore, in equation (11), $\mathbf{k}(\omega) \equiv R \cdot \mathbf{k}(\omega)_{\text {Flat Channel }}$ where $\mathbf{k}(\omega)_{\text {Flat Channel }}$ is the permeability of a fracture modeled as an open, parallel, flat channel. Biot-Willis coefficient $\alpha$ of the fracture is assumed to be 1 .

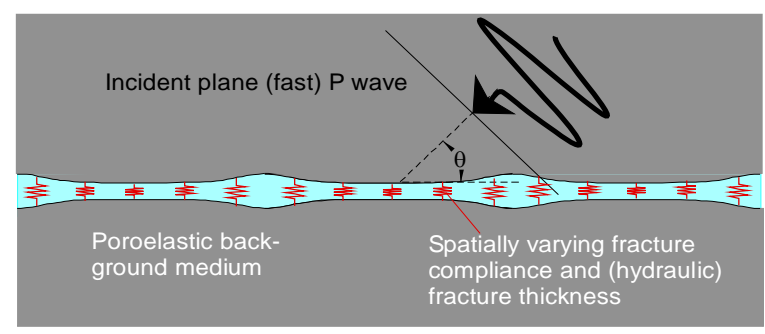

Figure 1: Wave scattering simulation for a periodic, heterogeneous, one-dimensional fracture 


\section{Poroelastic Heterogeneous Fracture Model}

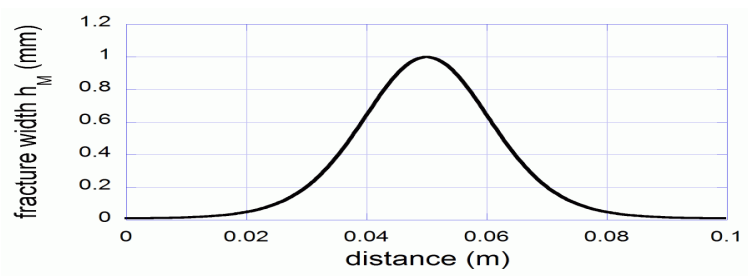

Figure 2: Hydraulic fracture width profile for a single period

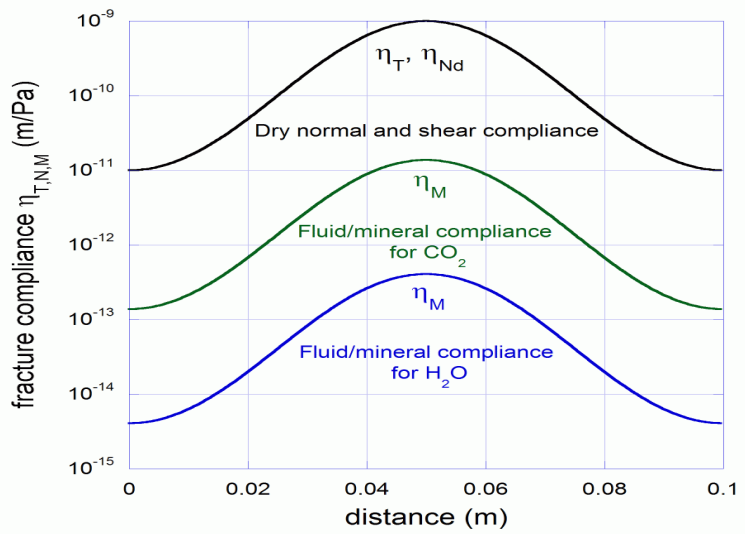

Figure 3: Fracture compliance profiles

\section{Water and super-critical $\mathrm{CO}_{2}$ saturated fracture}

First, we examine the case when the fracture is saturated by water (density $=992 \mathrm{~kg} / \mathrm{m}^{3}$, bulk modulus $=2.46 \mathrm{GPa}$, viscosity $=0.50 \mathrm{cP}$, at $\mathrm{T}=55^{\circ} \mathrm{C}$ and $\mathrm{P}=15 \mathrm{MPa}$ ) (Figure 4). In this case, the permeability of the background affects the reflection of normally incident fast $\mathrm{P}$ waves significantly. However, the permeability of the fracture itself has no visible impact on the scattering of fast $\mathrm{P}$ waves, for both normal and oblique $\left(45^{\circ}\right)$ incidence cases.

When the fluid within the fracture is substituted by supercritical $\mathrm{CO}_{2}$ (density $=653 \mathrm{~kg} / \mathrm{m}^{3}$, bulk modulus $=0.0726 \mathrm{GPa}$, viscosity $=0.05 \mathrm{cP}$ ), the scattering behavior becomes quite different for the low-permeability background cases (Figure 5). Instead of monotonic increases in the reflection coefficient amplitudes with frequency, the frequency response curves exhibit a small peak at a transition frequency depends on the fracture permeability. This transition frequency does not seem to shift for the different angles of incidence.

\section{High and Low-frequency-limit behavior of low background-permeability fractures}

In the high-frequency limit, the fluid in the fracture is not allowed to move. In this case, the $(4,4)$ term in the compliance matrix in equation (8) becomes 


\section{Poroelastic Heterogeneous Fracture Model}

$$
\alpha^{2} \eta_{N d}+\eta_{M}+(1 / i \omega) \nabla^{(2)} \cdot\left(\varsigma(\omega) \nabla^{(2)}\right) \rightarrow \alpha^{2} \eta_{N d}+\eta_{M}
$$

In the low-frequency limit, the fluid has enough time to flow within the fracture and relax the induced pressure differences. In this case, the fluid/solid compliance $\eta_{M}$ can be further replaced by its spatial average $\left(\eta_{M} \rightarrow \bar{\eta}_{M}\right)$.

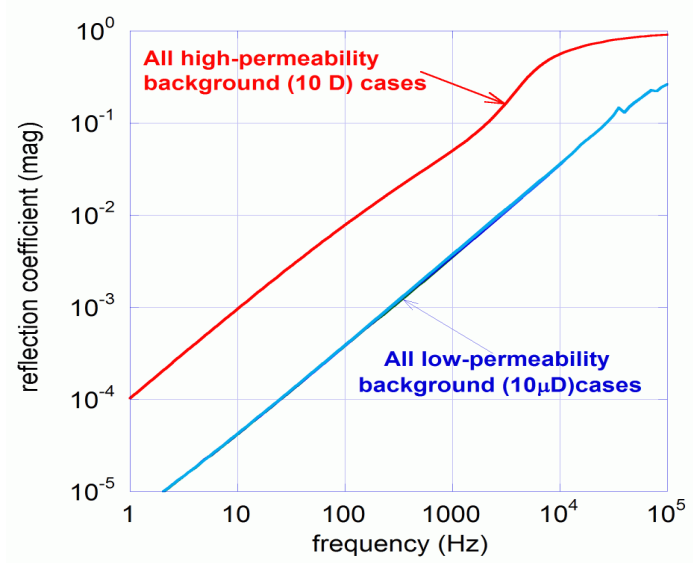

(a) Normal incidence

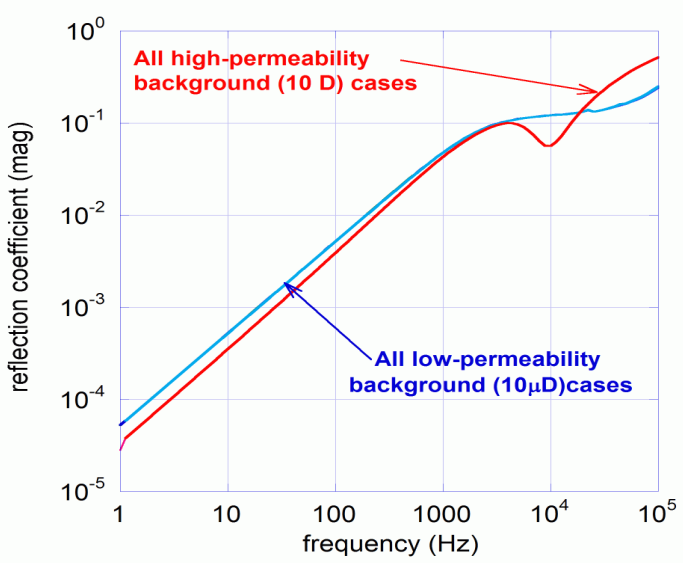

(b) $45^{\circ}$ incidence

Figure 4: Reflection coefficient amplitudes of fast $\mathrm{P}$ waves for a water-saturated fracture. $\mathrm{R}=1 / 1000-1$ indicate reduction factors for the reference fracture permeability given for an open fracture with a given local width.

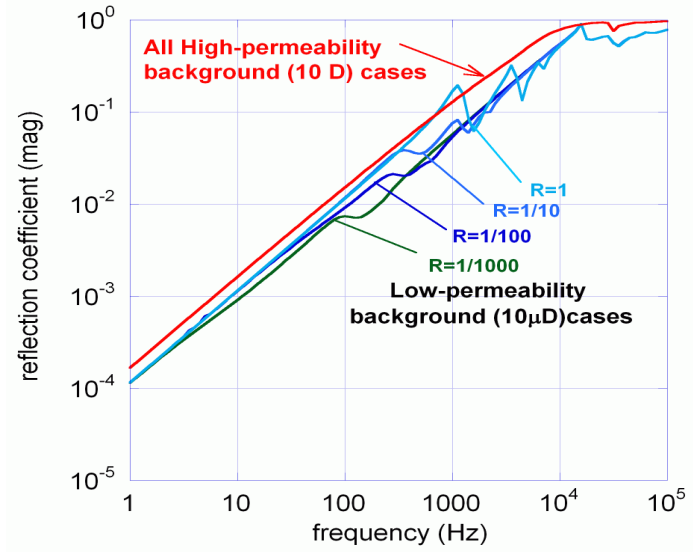

(a) Normal incidence

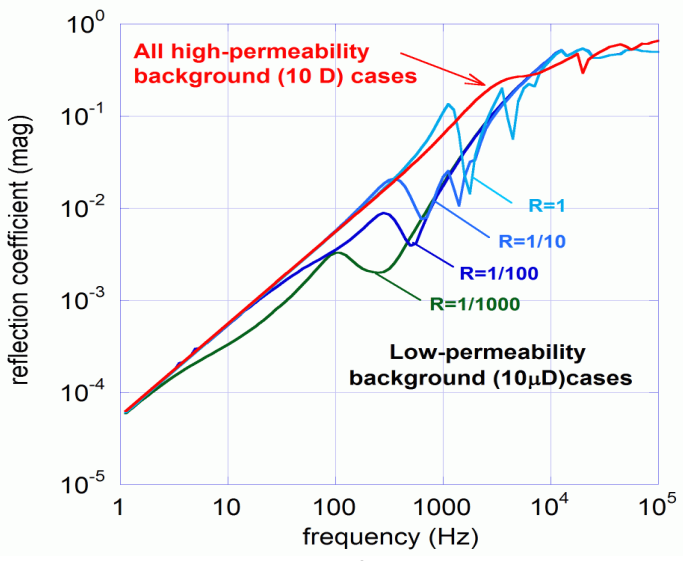

(b) $45^{\circ}$ incidence

Figure 5: Reflection coefficient amplitudes of fast $\mathrm{P}$ waves for a super-critical $\mathrm{CO}_{2}$ saturated fracture. $R=1 / 1000-1$ indicate reduction factors for the reference fracture permeability given for an open fracture with a given local width. 


\section{Poroelastic Heterogeneous Fracture Model}

Figures 6 shows both low and high-frequency limits of fast P-wave refection amplitudes. From Figure $6 b$, there is a significant difference in the reflection amplitudes between the low and highfrequency responses for the $\mathrm{CO}_{2}$-saturated fracture. Permeability of a fracture determines the transition frequency at which the behavior of the fracture changes between the two limits. In contrast, the two limiting responses are very similar for a water- saturated fracture (Figure 6a). Because of this, the permeability difference does not affect the scattering response.

\section{Conclusions}

We extended the Schoenberg's Linear Slip Interface model to a heterogeneous poroelastic fracture. The new model contains fracture permeability in the plan-parallel direction. From numerical simulations, we found that the direct impact of the fracture permeability can be seen in the frequency response of reflection amplitudes (for fast $\mathrm{P}$ waves). For a supercritical $\mathrm{CO}_{2}$-filled fracture, transition from the low-frequency response to high-frequency response can be seen as a peak in the response. However, a fracture filled with water exhibited no significant differences for a wide range of fracture permeability values.

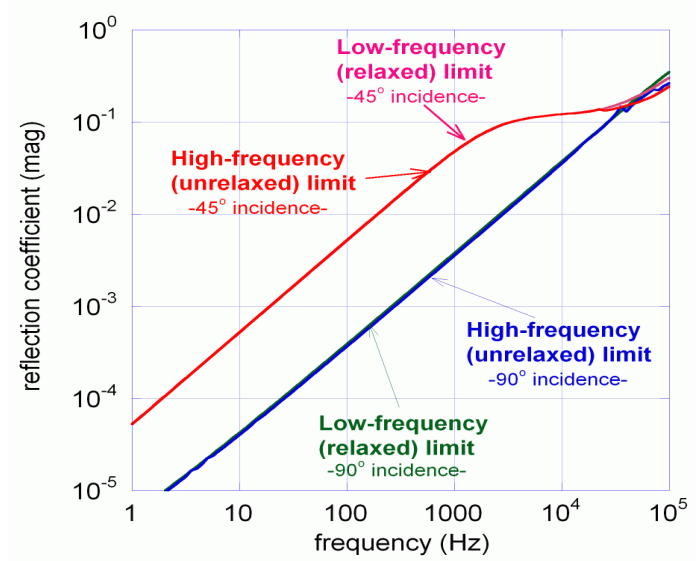

(a) Water-filled fracture

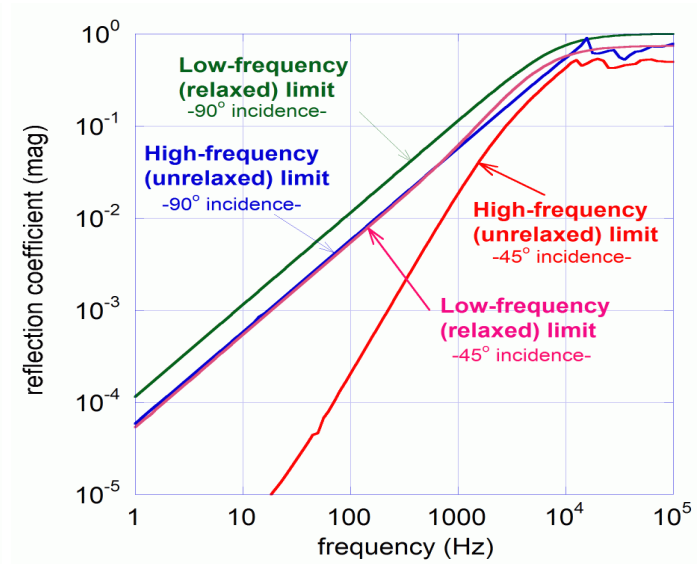

(b) $\mathrm{CO}_{2}$-filled fracture

Figure 6: High and low-frequency limit behavior of water and supercritical $\mathrm{CO}_{2}$-filled fractures. Compare to the latter, the both limiting cases for the water-saturated fractures are nearly identical (for each incidence angle), resulting in little sensivity of the seismic reflection to the fracture permeability. 


\section{Poroelastic Heterogeneous Fracture Model}

\section{Acknowledgments}

This research has been supported by the Office of Science, Office of Basic Energy Sciences, Division of Chemical Sciences of the U.S. Department of Energy under Contract No. DE-AC02$05 \mathrm{CH} 11231$.

\section{References}

Bakulin, A. andMolotkov, L., 1997, Poroelastic medium with fractures as limiting case of stratified poroelastic medium with thin and soft Biot layers: In $67^{\text {th }}$ Annual International Meeting, SEG, Expanded Abstracts, 1001-1004.

Biot, M.A., 1956, Theory of elastic waves in a fluid-saturated porous solid. II High frequency range: J. Acoust. Soc. Am., 28, 179-191.

Coates, R.T. and Schoenberg, M., 1995, Finite-difference modeling of faults and fractures: Geophysics, 60, 1514-1526.

Dvorkin, J., Nolen-Hoeksema, R., and Nur, A., 1994, The squirt-flow mechanism: macroscopic description: Geophysics, 59, 428-438.

Hsu, C.-J. and Schoenberg, M., 1993, Elastic waves through a simulated fractured medium: Geophysics, 58, 964-977.

Myer, L.R., Hopkins, D., and Cook N.G.W., 1990, Effect of contact area of an interface on acoustic wave transmission: In Proc. $26^{\text {th }}$ US Symposium on Rock Mechanics, 549-556.

Nakagawa, S. 2009. Poroelastic modeling of fracture-seismic wave interaction: Int. J. of the $J C R M, 4(2), 5-14$.

Nakagawa, S., Nihei, K.T., and Myer, L.R., 2004, Plane wave solution for elastic wave scattering by a heterogeneous fracture: J. Acoust. Soc. Am., 115, 2761-2773

Nakagawa, S. and Schoenberg, M., 2007, Poroelastic modeling of seismic boundary conditions across a fracture: J. Acoust. Soc. Am., 122, 831-847.

Pride, S.R. and Berrymann, J.G., 2003a, Linear dynamics of double-porosity and dualpermeability materials, I. Governing equations and acoustic attenuation: Phys. Rev.E, 68, DOI: 10.1103/PhysRevE.68.036603. 


\section{Poroelastic Heterogeneous Fracture Model}

Pride, S.R. and Berrymann, J.G., 2003b, Linear dynamics of double-porosity and dualpermeability materials, II. Fluid transport equation: Phys. Rev.E, 68, DOI: 10.1103/PhysRevE.68.036604.

Pyrak-Nolte, L., Cook, N.G.W., and Myer, L.R., 1990, Transmission of seismic waves across single natural fractures: J. Geophys. Res., 95, 8516-8538.

Schoenberg, M., 1980, Elastic wave behavior across linear slip interfaces: J. Acoust Soc. Am., 68, 1516-1521. 